\title{
PRECONDITIONED CONJUGATE GRADIENT ALGORITHMS FOR NONCONVEX PROBLEMS WITH BOX CONSTRAINTS
}

\author{
R. Pytlak, ${ }^{1}$ and T. Tarnawski, ${ }^{2}$ \\ ${ }^{1}$ Faculty of Cybernetics, Military University of Technology, 00-908 Warsaw, Poland, rpyt- \\ lak@isi.wat.edu.pl ${ }^{2}$ Faculty of Cybernetics, Military University of Technology,00-908 Warsaw, \\ Poland,tarni@isi.wat.edu.pl
}

\begin{abstract}
The paper describes a new conjugate gradient algorithm for large scale nonconvex problems with box constraints. In order to speed up the convergence the algorithm employs a scaling matrix which transforms the space of original variables into the space in which Hessian matrices of functionals describing the problems have more clustered eigenvalues. This is done efficiently by applying limited memory BFGS updating matrices. Once the scaling matrix is calculated, the next few iterations of the conjugate gradient algorithms are performed in the transformed space. The box constraints are treated by the projection as previously used in $[R$. Pytlak, The efficient algorithm for large-scale problems with simple bounds on the variables, SIAM J. on Optimization, Vol. 8, 532-560, 1998]. We believe that the preconditioned conjugate gradient algorithm gives more flexibility in achieving balance between the computing time and the number of function evaluations in comparison to a limited memory BFGS algorithm. The numerical results show that the proposed method is competitive to L-BFGS-B procedure.
\end{abstract}

keywords: bound constrained nonlinear optimization problems, conjugate gradient algorithms, quasi-Newton methods.

\section{Introduction}

In this paper we consider algorithms for the problem:

$$
\begin{gathered}
\min _{x \in \mathcal{R}^{n}} f(x) \\
\text { s. t. } l \leq x \leq u,
\end{gathered}
$$

where $l, u \in \mathcal{R}^{n}$.

In [9] (see also [4]) a new family of conjugate gradient algorithms has been introduced whose direction finding subproblem is given by

$$
d_{k}=-\operatorname{Nr}\left\{g_{k},-\beta_{k} d_{k-1}\right\}
$$

Please use the following format when citing this chapter:

Author(s) [insert Last name, First-name initial(s)], 2006, in IFIP International Federation for Information Processing, Volume 199, System Modeling and Optimization, eds. Ceragioli F., Dontchev A., Furuta H., Marti K., Pandolfi L., (Boston: Springer), pp. [insert page numbers]. 
where $\operatorname{Nr}\{a, b\}$ is defined as the point from a line segment spanned by the vectors $a$ and $b$ which has the smallest norm, i.e.,

$$
\|\operatorname{Nr}\{a, b\}\|=\min \{\|\lambda a+(1-\lambda) b\|: 0 \leq \lambda \leq 1\},
$$

$\|\cdot\|$ is the Euclidean norm and $g_{k}=\nabla f\left(x_{k}\right)$.

Notice that if $\beta_{k}=1$ then we have the Wolfe-Lemaréchal algorithm ([7], [13]). In [9] it was shown that the Wolfe-Lemaréchal algorithm is in fact the Fletcher-Reeves algorithm when directional minimization is exact. Moreover, the sequence $\left\{\beta_{k}\right\}$ was constructed in such way that directions generated by (3) are equivalent to those provided by the Polak-Ribiére formula (under the assumption that directional minimization is exact). This sequence

$$
\beta_{k}=\frac{\left\|g_{k}\right\|^{2}}{\left|\left\langle g_{k}-g_{k-1}, g_{k}\right\rangle\right|}
$$

has striking resemblance to the Polak-Ribiére formula.

\section{General preconditioned conjugate gradient algorithm}

The idea behind preconditioned conjugate gradient algorithm is to transform the decision vector by linear transformation $D$ such that after the transformation the nonlinear problem is easier to solve. If $\hat{x}$ is transformed $x$ :

$$
\hat{x}=D x
$$

then our minimization problem will become

$$
\min _{\hat{x}}\left[\hat{f}(\hat{x})=f\left(D^{-1} \hat{x}\right)\right]
$$

and for this problem the search direction will be defined as follows

$$
\hat{d}_{k}=-\operatorname{Nr}\left\{\nabla \hat{f}\left(\hat{x}_{k}\right),-\hat{\beta}_{k} \hat{d}_{k-1}\right\}
$$

Notice that

$$
\nabla \hat{f}(\hat{x})=D^{-T} \nabla f(\hat{x})
$$

therefore we can write

$$
\hat{d}_{k}=-\operatorname{Nr}\left\{D^{-T} \nabla f\left(D^{-1} \hat{x}_{k}\right),-\hat{\beta}_{k} \hat{d}_{k-1}\right\} .
$$

If we multiply both sides of (10) by $D^{-1}$ we will get

$$
d_{k}=-\lambda_{k} D^{-1} D^{-T} \nabla f\left(x_{k}\right)+\left(1-\lambda_{k}\right) \hat{\beta}_{k} d_{k-1} .
$$

where $0 \leq \lambda_{k} \leq 1$ and either

$$
\hat{\beta}_{k}=1
$$


for the Fletcher-Reeves version, or

$$
\hat{\beta}_{k}=\frac{\left\|\hat{g}_{k}\right\|^{2}}{\left|\left\langle\hat{g}_{k}-\hat{g}_{k-1}, \hat{g}_{k}\right\rangle\right|}=\frac{g_{k}^{T} D^{-1} D^{-T} g_{k}}{\left|\left(g_{k}-g_{k-1}\right)^{T} D^{-1} D^{-T} g_{k}\right|}
$$

for the Polak-Ribiere version.

The equation (11) can be stated as

$$
d_{k}=-\lambda_{k} H \nabla f\left(x_{k}\right)+\left(1-\lambda_{k}\right) \beta_{k} d_{k-1} .
$$

where $H=D^{-1} D^{-T}$. This suggests that $D$ should be chosen in such a way that $D^{T} D$ is an approximation to $\nabla_{x x}^{2} f(\bar{x})$ where $\bar{x}$ is a solution of problem (1).

Moreover, if $D$ is an upper triangular matrix then at each iteration of the algorithm we will have to solve the system of linear equations

$$
D^{T} \hat{g}_{k}=g_{k}, \quad D d_{k}=\hat{d}_{k} .
$$

It is worthwhile to notice that the following holds (see [11]):

$$
\left\langle g_{k}, d_{k}\right\rangle \leq-\left\|\hat{d}_{k}\right\|^{2} \text { and }\left\langle g_{k}, d_{k}\right\rangle=-\left\|\hat{d}_{k}\right\|^{2},
$$

if $0<\lambda_{k}<1$.

If box constraints (2) are present in our problem then we can tackle them by using the projection procedure proposed initially in [1] (see also [10]).

In the rest of the paper we consider, for the simplicity of presentation, the problems with simpler constraints $x \geq 0$. We define the set of indices $I_{k}^{+}$

$$
I_{k}^{+}:=\left\{i \in \overline{1, n}:\left(x_{k}\right)_{i} \leq \varepsilon_{k} \text { and } \nabla_{x_{i}} f\left(x_{k}\right)>0\right\},
$$

where $\left\{\varepsilon_{k}\right\}$ is such that $\varepsilon_{k}>0$ and

$$
\lim _{k \in K}\left\|x_{k}-P\left[x_{k}-\nabla f\left(x_{k}\right)\right]\right\|=0 \Leftrightarrow \lim _{k \in K} \varepsilon_{k}=0 .
$$

for any subsequence $\left\{x_{k}\right\}_{k \in K}$. Here, by $P[\cdot]$ we denote the projection operator on the set $\left\{x \in \mathcal{R}^{n}: l \leq z \leq u\right\}$ ([1]).

The sets $I_{k}^{+}$are used to modify the direction finding subproblem. Instead of solving problem (3) we find a new direction according to the rule

$$
d_{k}=-\operatorname{Nr}\left\{\nabla f\left(x_{k}\right),-\beta_{k} d_{k-1}^{+}\right\} .
$$

Here $d_{k-1}^{+}$is defined by

$$
\left(d_{k-1}^{+}\right)_{i}:=\left\{\begin{array}{ll}
\left(d_{k-1}\right)_{i} & \text { if } i \notin I_{k}^{+} \\
-\nabla_{x_{i}} f\left(x_{k}\right) / \beta_{k} & \text { if } i \in I_{k}^{+}
\end{array} .\right.
$$


To complete the description of the main components of our algorithm we have to show how to use scaling matrices in its preconditioned version. Having the set of indices $I_{k}^{+}$we do not scale variables corresponding to them and we apply general scaling to the others. Therefore, we use the scaling matrix of the form

$$
D_{k}=\left[\begin{array}{cc}
D & 0 \\
0 & I_{n_{k}}
\end{array}\right]
$$

where $n_{k}=\left|I_{k}^{+}\right|$.

In order to describe the line search procedure notice that the function $f\left(P\left[x_{k}+\right.\right.$ $\left.\left.\alpha d_{k}\right]\right)$ can be interpreted as a composition of two functions: the first one is Lipschitzian and the second one continuously differentiable. If we define

$$
x_{k}(\alpha)=x_{k}+d_{k}(\alpha), \quad \text { where }\left(d_{k}(\alpha)\right)_{i}:= \begin{cases}\alpha\left(d_{k}\right)_{i} & \text { if } \alpha \leq \alpha_{k}^{i} \\ \alpha_{k}^{i}\left(d_{k}\right)_{i} & \text { if } \alpha>\alpha_{k}^{i}\end{cases}
$$

and the breakpoints $\left\{\alpha_{k}^{i}\right\}_{1}^{n}$ are calculated as follows

$$
\alpha_{k}^{i}:=-\frac{\left(x_{k}\right)_{i}}{\left(d_{k}\right)_{i}}, \quad i=1, \ldots, n
$$

(assuming that if $\left(d_{k}\right)_{i} \geq 0$ then $\alpha_{k}^{i}=\infty$ ), then our directional minimization rule can be stated as follows.

$\mathbf{R} 1$ find the largest positive number $\alpha_{k}$ from the set $\left\{\theta^{k}: k=0,1, \ldots, \theta \in\right.$ $(0,1)\}$ such that for $\mu \in(0,1)$ we have

$$
f\left(x_{k}\left(\alpha_{k}\right)\right)-f\left(x_{k}\right) \leq-\mu\left[\alpha_{k} \sum_{i \notin I_{k}^{+}}\left(d_{k}\right)_{i}^{2}+\sum_{i \in I_{k}^{+}} \frac{\partial f\left(x_{k}\right)}{\partial x^{i}}\left[x_{k}^{i}-x_{k}^{i}\left(\alpha_{k}\right)\right],\right.
$$

Notice that in the rule $R 1$ we employ $d_{k}$ instead of $\hat{d}_{k}$ as (15) would imply. Since we assume that $D_{k}^{T} D_{k}$ are uniformly bounded from below and above (in the sense of condition (26)) there exist constants $0<c_{1}<c_{2}<+\infty$ such that $c_{1}\left\|d_{k}\right\| \leq\left\|\hat{d}_{k}\right\| \leq c_{2}\left\|d_{k}\right\|$. Thus the use of $d_{k}$ on the left on inequality in the rule $R 1$ is justified (it corresponds to appropriately choosing the coefficient $\mu$ ). It is worthwhile to observe that our descent direction rule allows for such inaccurate directional minimization search.

Our general algorithm is as follows:

Algorithm Parameters: $\mu \in(0,1), \epsilon>0,\left\{\hat{\beta}_{k}\right\}_{1}^{\infty}$, $\left\{D_{k}\right\}_{1}^{\infty}, D_{k} \in R^{n \times n}$ nonsingular matrix, $T \in R^{n \times n}$ nonsingular diagonal matrix.

Data: $x_{0}$ 
1) Set $k=0$, compute $d_{k}=-g_{k}$, go to Step 3).

2)Compute: $w_{k}=x_{k}-P\left[x_{k}-T \nabla f\left(x_{k}\right)\right], \varepsilon_{k}=\min \left(\varepsilon,\left\|w_{k}\right\|\right)$,

$$
\begin{aligned}
D_{k}^{T} \hat{g}_{k} & =g_{k} \\
\hat{d}_{k} & =-\operatorname{Nr}\left\{\hat{g}_{k},-\hat{\beta}_{k} \hat{d}_{k-1}^{+}\right\} \\
D_{k} d_{k} & =\hat{d}_{k}
\end{aligned}
$$

If $w_{k}=0$ then STOP.

3) Find a positive number $\alpha_{k}$ according to the rule $R 1$.

4) Substitute $P\left[x_{k}+\alpha_{k} d_{k}\right]$ for $x_{k+1}$, increase $k$ by one, go to Step 2 .

We can prove the lemma

Lemma 1 Assume that $x_{k}$ is a noncritical point, $D_{k}^{T} D_{k}$ is positive definite and $d_{k} \neq 0$ is calculated in Step 2 of Algorithm. Then there exists a positive $\alpha_{k}$ such that the condition stated in the rule $\mathrm{R} 1$ is

$$
\lim _{\alpha \rightarrow \infty} f\left(P\left[x_{k}+\alpha d_{k}\right]\right)=-\infty .
$$

To investigate the convergence of Algorithm we begin by providing a crucial lemma which requires the following assumptions.

Assumption 1 There exists $L<\infty$ such that

$$
\|\nabla f(y)-\nabla f(x)\| \leq L\|y-x\|
$$

for all $x, y$ from a bounded set.

Assumption 2 There exist $d_{l}, d_{u}$ such that $0<d_{l}<d_{u}<+\infty$ and

$$
d_{l}\|u\|^{2} \leq u^{T} D_{k}^{T} D_{k} u \leq d_{u}\|u\|^{2}
$$

for all $u \in \mathcal{R}^{n}$ and $k$.

LeMma 2 Suppose that Assumptions 1-2 hold, the direction $d_{k}$ is determined by (22)-(24) and the step-size coefficient $\alpha_{k}$ is calculated according to the rule R1. Then, for any bounded subsequence $\left\{x_{k}\right\}_{k \in K}$ either

$$
\lim _{k \in K}\left\|x_{k}-P\left[x_{k}+d_{k}\right]\right\|=0,
$$

or

$$
\lim _{k \in K}\left\|x_{k}-P\left[x_{k}-\nabla f\left(x_{k}\right)\right]\right\|=0 .
$$

For the convenience of future notations we assume that variables $(x)_{i}$ have been reordered in such a way that $d_{k}$ can be partitioned into two vectors $\left(d_{k}^{1}, d_{k}^{2}\right)$ where the first vector $d_{k}^{1}$ is represented by

$$
d_{k}^{1}:=\left\{\left(d_{k}\right)_{i}\right\}_{i \notin I_{k}^{+}} .
$$


The same convention applies to other vectors.

THEOREM 3 Suppose that Assumptions 1-2 are satisfied. Moreover, assume that for any convergent subsequence $\left\{x_{k}\right\}_{k \in K}$ whose limit is not a critical point

i) $\left\{\hat{\beta}_{k}\right\}$ is such that

$$
\liminf _{k \rightarrow \infty}\left(\hat{\beta}_{k}\left\|d_{k-1}^{1}\right\|\right) \geq \nu_{1} \liminf _{k \rightarrow \infty}\left\|\nabla^{1} f\left(x_{k}\right)\right\|
$$

where $\nu_{1}$ is some positive constant,

ii) there exists a number $\nu_{2}$ such that $\nu_{2}\left\|D_{k}^{-T}\right\|_{2}\left\|D_{k-1}\right\|_{2} \in(0,1)$ and

$$
\left\langle\nabla^{1} f\left(x_{k}\right), d_{k-1}^{1}\right\rangle \leq \nu_{2}\left\|\nabla^{1} f\left(x_{k}\right)\right\|\left\|d_{k-1}^{1}\right\| \text {, whenever } \lambda_{k} \in(0,1) \text {. }
$$

Then $\lim _{k \rightarrow \infty} f\left(x_{k}\right)=-\infty$, or every accumulation point of the sequence $\left\{x_{k}\right\}_{0}^{\infty}$ generated by Algorithm is a critical point.

Our global convergence result is as follows.

THEOREM 4 Suppose that Assumptions 1-2 are satisfied. Then Algorithm generates $\left\{x_{k}\right\}$ such that every accumulation point of $\left\{x_{k}\right\}$ satisfies necessary optimality conditions for problem (1)-(2) provided that:

i) $\hat{\beta}_{k}$ is given by

$$
\hat{\beta}_{k}=\frac{\|\left.\nabla^{1} \hat{f}\left(\left(\hat{x}_{k}^{1}, \hat{x}_{k-1}^{2}\right)\right)\right|^{2}}{\left|\left\langle\nabla^{1} \hat{f}\left(\left(\hat{x}_{k}^{1}, \hat{x}_{k-1}^{2}\right)\right)-\nabla^{1} \hat{f}\left(\hat{x}_{k-1}\right), \nabla^{1} \hat{f}\left(\left(\hat{x}_{k}^{1}, \hat{x}_{k-1}^{2}\right)\right)\right\rangle\right|},
$$

ii) there exists $M<\infty$ such that $\alpha_{k} \leq M, \forall k$.

\section{Scaling matrices based on the compact representation of BFGS matrices}

In the previous section we showed that for a given nonsingular matrix $H^{-1}=$ $D^{T} D$ the preconditioned conjugate gradient algorithm is globally convergent. The use of constant scaling matrix is likely to be inefficient since the function $f$ we minimize is nonlinear. Therefore, we are looking at the sequence of matrices $\left\{H_{k}\right\}$ such that each $H_{k}^{-1}$ is as close as possible to the Hessian $\nabla_{x x}^{2} f\left(x_{k}\right)$ and can be easily factorized as $D_{k}^{-1} D_{k}^{-T}$ where $D_{k}$ is a nonsingular matrix. We assume, for the simplicity of presentation, that $n_{k} \equiv 0$.

In the paper we present the preconditioned conjugate gradient algorithm based on the BFGS updating formula. To this end we recall compact representations of quasi-Newton matrices described in [8]. Suppose that the $k$ vector 
pairs $\left\{s_{i}, y_{i}\right\}_{i=0}^{k-1}$ satisfy $s_{i}^{T} y_{i}>0$ for $i=k-m-1, \ldots, k-1$. If we assume that $B_{0}=\gamma_{k} I$ and introduce matrices $M_{k}=\left[\gamma_{k} S_{k} Y_{k}\right]$,

$$
S_{k}=\left[s_{k-m-1}, \ldots, s_{k-1}\right], Y_{k}=\left[y_{k-m-1}, \ldots, y_{k-1}\right]
$$

where $s_{i}=x_{i+1}-x_{i}$ and $y_{i}=g_{i+1}-g_{i}$, then LBFGS approximation to the Hessian matrix is

$$
B_{k}=\gamma_{k} I-M_{k} W_{k} M_{k}^{T}
$$

and $W_{k} \in \mathcal{R}^{m \times m}$ is nonsingular ([3]).

In order to transform the matrix $B_{k}$ to the form $D_{k}^{T} D_{k}$ we do the $\mathrm{QR}$ factorization of the matrix $M_{k}^{T}$ :

$$
M_{k}^{T}=Q_{k} R_{k}
$$

where $Q_{k}$ is $n \times n$ orthogonal matrix and $R_{k}$ the $n \times m$ matrix which has zero elements except the elements constituting the upper $m \times m$ submatrix. Taking into account that $Q_{k}^{T} Q_{k}=I$ we can write (33) as

$$
B_{k}=Q_{k}^{T}\left[\gamma_{k} I-R_{k}^{T} W_{k} R_{k}\right] Q_{k} .
$$

Notice that the matrix $R_{k}^{T} W_{k} R_{k}$ has zero elements except those lying in the upper left $m \times m$ submatrix. We denote this submatrix by $T_{k}$ and we can easily show that it is a positive definite matrix. If we compute the Cholesky decomposition of the matrix $\gamma_{k} I_{k}-T_{k}, \gamma_{k} I_{k}-T_{k}=C_{k}^{T} C_{k}$ then eventually we come to the relation

$$
B_{k}=Q_{k}^{T} F_{k}^{T} F_{k} Q_{k}
$$

with

$$
F_{k}=\left[\begin{array}{cc}
C_{k} & 0 \\
0 & \sqrt{\gamma_{k}} I_{n-k}
\end{array}\right]
$$

The desired decomposition of the matrix $B_{k}$ is thus given by

$$
B_{k}=D_{k}^{T} D_{k}, D_{k}=F_{k} Q_{k}
$$

where the matrix $D_{k}$ is nonsingular provided that $s_{i}^{T} y_{i}>0$ for $i=k-m-1$, $\ldots, k-1$. Notice that the matrix $Q_{k}$ does not have to be stored since it can be easily evaluated from the Householder vectors which have been used in the QR factorization.

Recall the relation (14) which now can be written as

$$
Q_{k}^{T} F_{k}^{T} \hat{g}_{k}=g_{k}, \quad F_{k} Q_{k} d_{k}=\hat{d}_{k} .
$$




\section{Scaling matrices - the reduced Hessian approach}

The approach is based on the limited memory reduced Hessian method proposed by Gill and Leonard ([5],[6], see also [12])

Suppose that $\mathcal{G}_{k}=\operatorname{span}\left\{g_{0}, g_{1}, \ldots, g_{k}\right\}$ and let $\mathcal{G}_{k}^{\perp}$ denote the orthogonal complement of $\mathcal{G}_{k}$ in $\mathcal{R}^{n}$. If $B_{k} \in \mathcal{R}^{n \times r_{k}}$ have columns that define the basis of $\mathcal{G}_{k}$ and

$$
H_{k}=Q_{k} T_{k}
$$

is the $\mathrm{QR}$ decomposition of $B_{k}$ then

$$
Q_{k}^{T} B_{k} Q_{k}=\left(\begin{array}{cc}
Z_{k}^{T} B_{k} Z_{k} & 0 \\
0 & \sigma I_{n-r_{k}}
\end{array}\right)
$$

where $Q_{k}=\left(Z_{k} W_{k}\right)$ and range $\left(B_{k}\right)=\operatorname{range}\left(Z_{k}\right)$. (40) follows from the theorem which was stated, among others, in [6]:

THEOREM 5 Suppose that the BFGS method is applied to a general nonlinear function. If $B_{0}=\sigma I_{n}$ and

$$
B_{k} d_{k}=-g_{k},
$$

then $d_{k} \in \mathcal{G}_{k}$ for all $k$. Furthermore, if $z \in \mathcal{G}_{k}$ and $w_{k} \in \mathcal{G}_{k}^{\perp}$, then $B_{k} z \in \mathcal{G}_{k}$ and $B_{k} w=\sigma w$.

From (40) we have

$$
B_{k}=D_{k}^{T} D_{k}=Q_{k}\left(\begin{array}{cc}
Z_{k}^{T} B_{k} Z_{k} & 0 \\
0 & \sigma I_{n-r_{k}}
\end{array}\right) Q_{k}^{T}
$$

Therefore, it follows that we can take as $D_{k}$ :

$$
D_{k}=\left(\begin{array}{cc}
R_{k} & 0 \\
0 & \sqrt{\sigma} I_{n-r_{k}}
\end{array}\right) Q_{k}^{T}=G_{k} Q_{k}^{T}
$$

At every iteration we have to solve equations

$$
Q_{k} G_{k}^{T} \hat{g}_{k}, \quad=g_{k} G_{k} Q_{k}^{T} d_{k}=\hat{d}_{k} .
$$

Solving these equations requires multiplication of vectors in $\mathcal{R}^{n}$ by the orthogonal matrix $Q_{k}$ (or $Q_{k}^{T}$ ), and this can be achieved by the sequence of $m$ multiplications of the Householder matrices $H_{k}^{i}, i=1, \ldots, m$ such that $Q_{k}=H_{k}^{1} H_{k}^{2} \cdots H_{k}^{m}$. The cost of these multiplications is proportional to $n$. Furthermore, we have to solve the set on $n$ linear equations with the upper triangular matrix $G_{k}$, or its transpose. 


\section{Numerical experiments}

In order to verify the effectiveness of our algorithm we have tested it on problems from the CUTE collection ([2]). We tried it on problems with various dimension although its application is recommended for solving large scale problems.

Algorithm has been implemented in $\mathrm{C}$ on Intel PC under Linux operating system. We compared our algorithm with the L-BFGS-B code which is the benchmark procedure for problems with box constraints for which evaluating the Hessian matrix is too expensive. L-BFGS-B code was used with the parameter $m=5$ and we applied $m=5$ and we recalculated matrices $D_{k}$ every five iterations in Algorithm. The stopping criterion was $\|\nabla f(x)\| / \max (1,\|x\|) \leq$ $10^{-7}$. We used the scaling matrices as described in Section 3.

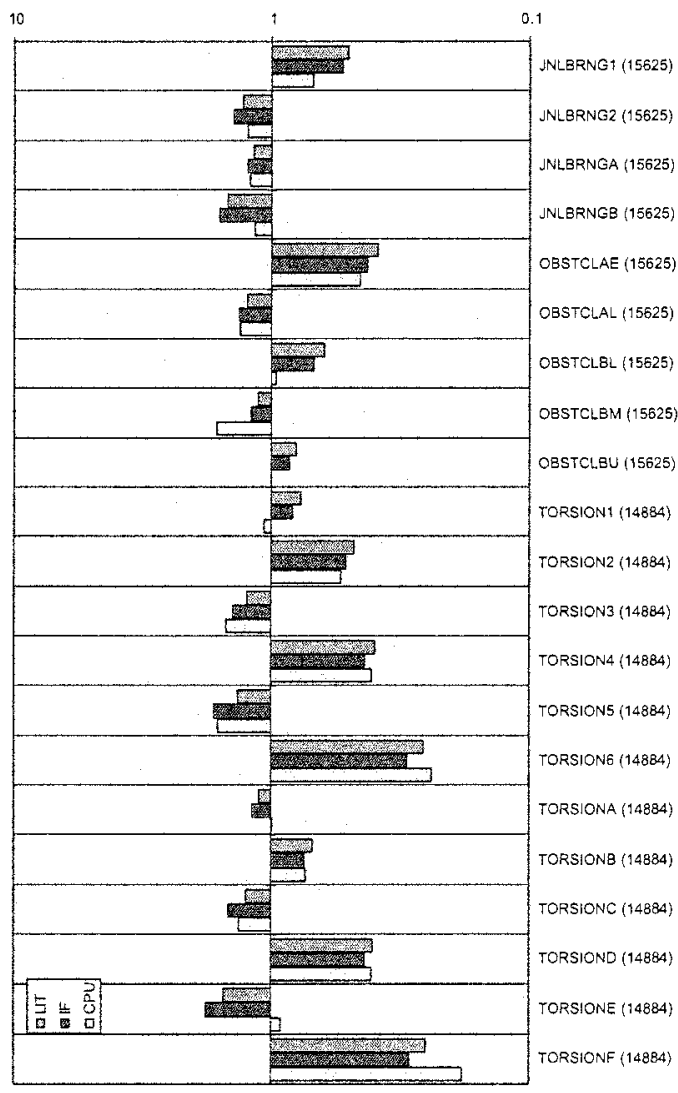

Figure 1. Performance comparison of Algorithm against the L-BFGS-B code. 
The performance comparison of Algorithm is given in Figure 1. where we compare it with the code L-BFGS-B presented in [14]. For each problem the bars represent the ratio of the number of iterations (LIT), number of function evaluations (IF) and computing time (CPU) needed by the Algorithm divided by those from the executions of the L-BFGS-B code. Therefore values above one testify in favor of the L-BFGS-B and below one - in favor of our algorithm.

\section{References}

[1] D.P. Bertsekas, Projected Newton methods for optimization problems with simple constraints. SIAM J. Control and Optimiz. 20:221-245. 1982.

[2] I. Bongartz, A.R. Conn, N.I.M. Gould, Ph.L. Toint, CUTE: Constrained and Unconstrained Testing Environment. Research Report RC 18860, IBM T.J. Watson Research Center, Yorktown Heights, NY, USA, 1994.

[3] R. Byrd, J. Nocedal, R. B. Schnabel, Representations of quasi-Newton matrices and their use in limited memory methods. Technical Report NAM-03, 1996.

[4] Y. Dai, Y. Yuan, Global convergence of the method of shortest residuals. Numerische Mathematik 83:581-598, 1999.

[5] P.E. Gill, M.W. Leonard, Reduced-Hessian methods for unconstrained optimization. SIAM J. Optimiz. 12:209-237, 2001.

[6] P.E. Gill, M.W. Leonard, Limited-memory reduced hessian methods for large-scale unconstrained optimization. SIAM J. Optimiz. 14:380-401, 2003.

[7] C. Lemaréchal, An Extension of Davidon methods to nondifferentiable Problem. In Mathematical Programming Study 3. North-Holland, Amsterdam, 1975.

[8] J. Nocedal, S.J. Wright, Numerical optimization. Springer-Verlag, New York, 1999.

[9] R. Pytlak, On the convergence of conjugate gradient algorithms. IMA Journal of Numerical Analysis. 14:443-460, 1994.

[10] R. Pytlak, An efficient algorithm for large-scale nonlinear programming problems with simple bounds on the variables. SIAM J. on Optimiz. 8:532-560, 1998.

[11] R. Pytlak, T. Tarnawski, The preconditione conjugate gradient algorithm for nonconvex problems. Research Report, Military University of Technology, Faculty of Cybernetics, N.-1, 2005.

[12] D. Siegel, Modifying the BFGS update by a new column scaling technique. Mathematical Programming. 66:45-78, 1994.

[13] P. Wolfe, A Method of Conjugate Subgradients for Minimizing Nondifferentiable Functions. In Mathematical Programming Study 3. North-Holland, Amsterdam, 1975.

[14] C. Zhu, R.H. Byrd, P. Lu, J. Nocedal, Algorithm 778: L-BFGS-B, FORTRAN subroutines for large scale bound constrained optimization. ACM Transactions on Mathematical Software. 23:550-560, 1997. 